\title{
«Ma nient mì podi dì del sentiment». Il canzoniere stilnovista di Franco Loi
}

\author{
Pietro Montorfani \\ Archivio storico della Città di Lugano
}

\begin{abstract}
Si offre una lettura della raccolta Verna (1997) del poeta milanese Franco Loi, un piccolo canzoniere di matrice stilnovista che segue da lontano le orme di Dante (e di alcune sue riletture novecentesche) per approdare assieme a lui al superamento di quella poetica.
\end{abstract}

Keywords: Franco Loi, Stilnovo, dialetto milanese.

Nella lunga esperienza poetica di Franco Loi (1930), iniziata nei primi anni Settanta e ancora molto prolifica nonostante l'età avanzata, la breve raccolta Verna si situa grosso modo a metà strada. Per la sua posizione cronologica, oltre che per le sue peculiarità di stile e contenuto, potrebbe venire facilmente eletta a emblema di un'intera carriera, tanto più che pare essere uno dei punti di più stretto dialogo con la tradizione (anche delle origini), caratteristica che la accomuna a uno dei vertici della letteratura milanese: Carlo Porta che, tra il serio e il faceto, ma più il primo del secondo, fra il I80I e il I805 reinterpreta alcuni frammenti dei primi canti dell'Inferno dantesco con esiti di una vitalità e di un'originalità ancora oggi sorprendenti («gh'emm miss el segn, e s'ciavo!» per «quel giorno più non vi leggemmo avante», e simili intuizioni capaci di tenere il passo dell'archetipo)'.

Che il dialetto non potesse essere soltanto lingua viva, ma dovesse subire in qualche misura una rielaborazione formale, retorica, «letteraria» nel senso più alto del termine, era un destino inscritto nella biografia dell'autore. Nato a Genova da padre sardo e madre emiliana di Colorno, Franco Loi apprese il milanese in gioventù, nel quartiere meneghino di Casoretto, nei pressi di Lambrate, e ne fece il suo principale mezzo espressivo a seguito di una sincera quanto misteriosa infatuazione, l'incontro con una lingua "più attenta ai suoni che ai significati» (Tessa) e, anche per questo, profondamente inscritta in una precisa dimensione geografica e sociale:

Quand'ero bambino o ragazzo, camminavo per le strade e sentivo il milanese. Forse non avrei mai scritto in milanese, se non ci fosse stata questa

I Si cita da Porta 2000: 226-239 (Canto I), 672-677 (Canto II), 678-679 (Canto III), 682-686 (Canto V), 687-696 (Canto VII). 
simbiosi con la musica della città. C'era anche chi parlava in italiano, specialmente nel dopoguerra, ma ovunque sentivo la voce lombarda, con la sua ironia, quei toni un po' rauchi, anche nello straparlare italiano. [...] Io, poi, abitavo in una zona di immigrati e tra noi si parlava italiano, ma il tono, la forma del dire e del sentire, tra noi bambini, erano già quelli del milanese (Loi 20I0: 77$)^{2}$.

L'esordio in poesia, frutto di una vocazione potente maturata in pochi mesi nell'estate del I970, avvenne tre anni più tardi sull'Almanacco dello Specchio Mondadori, favorito da Vittorio Sereni e Dante Isella. È l'inizio di un percorso costellato di una trentina di titoli, da Stròlegh (I975) a L'angel (I98I), da Bach (1986) a Liber (1988) ad Aria de la memoria (2005), su su fino al recentissimo Voci d'un vecchio cantare, pubblicato da Il Ponte del Sale nel 20I7. Editori nazionali e piccole case editrici milanesi come Scheiwiller e Crocetti si sono avvicendati nella pubblicazione dei suoi versi, e nemmeno sono mancate plaquettes a latitudini insolite per la poesia lombarda, dalla pugliese Manni alla romana Empiria (proprio per Verna), segno non soltanto di una fama oramai consolidata, ma anche di una rinnovata attenzione, comune a tutta la Penisola, per le letterature regionali in dialetto.

La raccolta Verna («Inverno») apparve nel 1997 quale titolo inaugurale di una collana, «Poesia \& variazioni», diretta da Daniela Attanasio. Rispettando le consegne di quella sede editoriale, Loi propose un libro composto di due parti - almeno in apparenza - nettamente distinte: un piccolo canzoniere d'amore di 26 pezzi (che per comodità, d'ora innanzi, richiameremo con le sigle $V_{1}, V_{2}, V_{3}, \ldots, V_{26}$ ) e tre libere versioni dialettali di altrettanti passi della letteratura mondiale, da Orazio (l'epodo XVI), Calderón de la Barca (il primo monologo di Sigismondo da La vida es sueño) e Vicente Aleixandre (Al amor), di cui Loi fornisce, per contrasto, anche una più letterale traduzione italiana a piè di pagina. L'accostamento di questi tre pezzi e, più in generale, delle due parti che compongono il libro è sintomo dell'ambizione che deve aver dettato queste pagine, davvero il prodotto di un fine lavoro di cesello attorno a temi capitali della tradizione letteraria (l'amore, la morte, il corpo, l'esperienza dell'altro, il senso del tempo che passa, il destino di ciascuno) e di un libero adattamento di stilemi e codici retorici giunti fino a noi da un illustre passato. Il rischio - i lettori se ne avvedranno subito - è quello della maniera, del riutilizzo di una partitura usurata e perciò priva di reale energia espressiva; un rischio che Loi elude grazie a minimi aggiustamenti di tono che portano, pagina dopo pagina, verso dopo verso, a una sua personale verità, espressa con forza proprio all'altezza dello snodo $\left(\mathrm{V}_{24}-\mathrm{V}_{26}\right)$ che lega

2 Il rapporto con l'opera di Tessa, successivo alla prima vocazione poetica di Loi ma da subito molto solido, è indagato indirettamente anche nel volume da lui curato Milano. Lo sguardo di Delio Tessa, Milano, Edizioni Unicopli («Le Città Letterarie»), 2003. 
la prima e la seconda parte del libro, il canzoniere d'amore alle «variazioni» stimolate dalla proposta di Daniela Attanasio.
Sì, mì di dònn, l'amur, quj sò mister, tüsscoss me pias.. Scultàj, tucàj, parlàgh..
Me pias quel mòrvid durmì ind i sò brasc, sentì i sò man.. E pö sugnàj, me pias..
Sì, mì di dònn, el cör, quel delecâ
che par respira, l'umbra, i laver fregg..
Me pias fina quel nient che dent te resta
quan' che te bràscen e pö te tràn luntan.

Il componimento introduttivo della prima parte $\left(\mathrm{V}_{\mathrm{I}}\right)$, ripreso non per nulla anche nella quarta di copertina, è doppiamente programmatico: nell'esplicita dichiarazione del tema - la donna e i suoi misteri - come nel dispiegarsi di buona parte delle strategie retoriche che si ritroveranno nei venticinque pezzi successivi ${ }^{3}$. L'endecasillabo, innanzitutto, a cui Loi affida il passo lungo e ritmato del suo incedere, spesso in coppie alternate di tronchi e piani (in $V_{\text {I }}$ soltanto il penultimo verso, ma è esso stesso una costante, quasi una «regola del penultimo» che, in sede metrica, non pare essere del tutto casuale $)^{4}$. Il metro principe della tradizione letteraria italiana, di grande fortuna anche in ambito lombardo, si compatta in Verna in strutture monostrofiche prive di titoli (rarissimi in Loi, come pure i salti di riga) anche grazie al fitto richiamarsi di suoni lungo tutta la dorsale del testo, per mezzo di anafore (vv. I-5 e 2-7), rime interne («quel nient che dent», v. 7) e ripetizioni (due volte «dònn», quattro «pias»), ma anche allitterazioni, assonanze, consonanze: insomma l'intero campionario di quelle figure linguistiche che hanno quale esito comune la messa in evidenza della funzione poetica (Jakobson) in ogni testo letterario seriamente inteso.

Nello specifico, la monostrofa di VI si avvita circolarmente attorno al predicato «me pias», vero e proprio cuore sintattico e semantico della poesia, replicato quattro volte a quattro altezze diverse (vv. 2, 3, 4 e 7 ) in tre differenti sedi metriche: a metà strada del secondo e alla fine del quarto verso, mentre la seconda e la quarta replica costituiscono un'anafora perfettamente simmetrica, ad apertura del terzo verso di due quartine (introdotte entrambe da «Sì, mì di dònn», vv. I e 5). Le necessità della variatio impongono

3 Il testo compare, con altri tre pezzi della medesima raccolta $\left(V_{2}, V_{5}, V_{1} 8\right)$, nell'autoantologia Aria de la memoria. Poesie scelte 1973-2002, pubblicata nel 2005.

4 Costante in tutta la prima parte del libro, una netta individuazione metrica del penultimo verso (perché irrelato o comunque estraneo allo schema rimico) è particolarmente evidente in $\mathrm{V}_{2}, \mathrm{~V}_{3}, \mathrm{~V}_{4}, \mathrm{VI}_{5}$, VI6, V24. Pensata forse per eludere la facile soluzione del distico finale a rima baciata, tale regola si interrompe significativamente soltanto nel pezzo conclusivo, sciogliendosi nell'evocativa eco di «gloria» in «storia». 
inoltre al modulo una disposizione sintattica alternata: nel primo e nel terzo caso si trova in fine di frase, posposto al soggetto che regge il predicato («l'amur, quj sò mister, tüsscoss» e «sugnàj»), mentre nelle altre occorrenze precede e inaugura un periodo di andamento rovesciato - sebbene, in realtà, secondo una dispositio più ovvia e tradizionale (l'italiano «piacere» non funziona come l'inglese «to like»). Tale attenzione alla dimensione spaziale, rafforzata a chiusura del secondo, del quarto e del sesto verso da due puntini di sospensione (il Gadda della Cognizione ne usava invece quattro), è naturalmente funzionale al messaggio, direi anzi strategica, se è vero che il plazer della poesia provenzale e poi stilnovista è la chiave d'accesso a questa riattualizzazione dialettale e tutta lombarda della poesia amorosa delle origini.

Al pari dei primi autori della tradizione romanza, anche per Loi l'amore è al contempo di natura corporale e spirituale, desiderio di tucà e sentì (le mani, le braccia, le labbra) ma anche sogno e mistero, quel niente che rimane nel cuore dopo la partenza dell'amata. Sull'antitesi tra corpo e spirito si fondano molti dei primi testi della raccolta; soprattutto $\mathrm{V}_{5}$ pare tutto giocato sulla giustapposizione, spaziale e semantica assieme, del toccare e del ridere, dentro un'atmosfera che, con la complicità della notte, prende la forma di un sogno che si nasconde dentro un altro sogno:

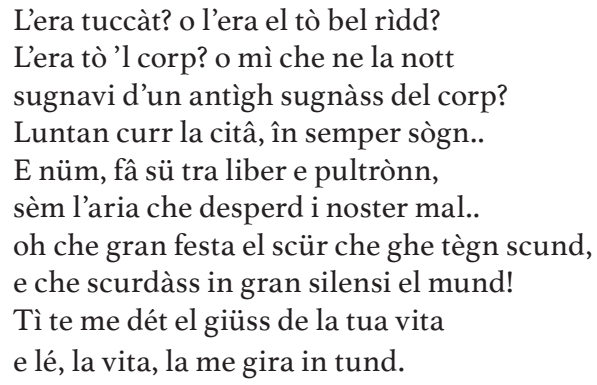

Seguendo i dettami del più consolidato codice stilnovista, anche la dimensione dello sguardo trova una sua costante applicazione nel canzoniere. Numerosissime sono infatti le occorrenze di vardà, varden, vardàt, vardàss: specie in $V_{2}, V_{3}, V_{6}, V_{7}$, $V_{10}$ («de quèl che me piaseva del vardà», v. 4), e ancora in $V_{I 6}$, VI9 e $V_{21}$ («E alura vardi i dònn, e me par sensa / che g'abia de vardàj per 'namuràss», vv. 4-5). L'atto stesso del guardare e del guardarsi, riflesso nell'oggetto del desiderio, può spingersi metaforicamente fino alle più estreme conseguenze: «Sé l'è che me muriva nel vardàss?» (VI9, v. 5). Se meno incisivo è il ricorso al verbo vedere, comunque utilizzato a sprazzi (in $\mathrm{V}_{4}$, $\mathrm{V}_{\mathrm{I}} 6$ e soprattutto in $\mathrm{V}_{25}$, ma con diversa accezione, di cronaca in presa diretta: «Vu 'n gir e vedi tocch / d'una Milan de gent che va a cassü», vv. I-2), 
decisamente preponderante - ma non poteva essere altrimenti - è la presenza di parere, vero predicato-simbolo, ai nostri occhi di moderni, della poetica stilnovista, soprattutto a partire dalla magistrale lettura continiana di Tanto gentile (Contini I970: I6I-I68). Nel canzoniere di Loi il termine ricorre quasi sempre nell'accezione più ovvia di «sembrare» (due volte ad esempio in $\mathrm{V}_{7}$ : «Oh dòna, ch'a vardàt te pàret vera / [...] / quèl'àves due la vita par se trova», vv. I e 7) e praticamente mai in quella di «apparire» segnalata da Contini; risultano comunque evidenti le tracce mnemoniche - lessicali, ritmiche e sintattiche insieme - lasciate in Loi dalla lettura del testo dantesco, quasi una matrice nel cui calco siano stati fusi molti versi di queste liriche amorose:

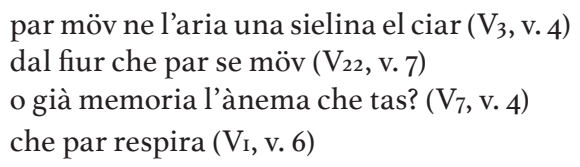

Passi che sembrano davvero mutuati dalla terzina conclusiva del sonetto dantesco:

$$
\begin{aligned}
& \text { e par che de la sua labbia si mova } \\
& \text { un spirito soave pien d'amore, } \\
& \text { che va dicendo a l'anima: Sospira. }
\end{aligned}
$$

Lungo il confine semantico tra il guardare (vedere, sembrare, apparire) e il sognare (esperienza notturna e diurna, ad occhi chiusi oppure aperti) si situa l'immagine dello specchio, attorno alla quale pure si dispongono alcuni dei pezzi più interessanti del libro: «Oh tì, Miranda, tra i linsö retort, / dòna ch'in tì se cerca el fögh del ver, / la mort che nel tò lett l'è 'n spegg cuj port» ( $\mathrm{V}_{4}$, vv. 8-IO, dove, oltre al nome della donna, che ha tutto per sembrare un ironico senhal sulla scia della cavalcantiana Chi è questa che vèn, ch'ogn'om la mira, nel nono verso ci sarà forse anche una lontana eco di Paradiso XXXIII); «mì sun sul, tì sula, e la memoria / ver la fa j ùmber e feng la veritâ, / d'un nient che resta, che traversa i sògn / e spègg nel spègg me lassa nel speggiàss» ( $\mathrm{V}_{7}$, vv. 9-I2). Quest'ultimo caso, tratto dal brano più pirotecnico e stilizzato dell'intera raccolta, pone sul tavolo una questione cruciale, cioè quella della verità e, di rimbalzo, del suo rapporto con il potere e l'efficacia del linguaggio letterario: «Oh dòna, ch'a vardàt te pàret vera, / bèj làver ch'aj mè laver san tucà, / l'è gioia o l'è 'n engann la mia parola» $\left(\mathrm{V}_{7}, \mathrm{vv}\right.$. I-4). Che Loi giunga - come Dante e, nei secoli successivi, un nutrito gruppo di poeti dietro di lui - ad ammettere il rischio dell'afasia è contemporaneamente il frutto di una scelta retorica e il cristallizzarsi di un'esperienza personale, a 
metà strada tra la cronaca di una vicenda amorosa e un più profondo interrogarsi sulle questioni ultime (metafisiche, religiose) 5 .

In un punto il gioco combinatorio, di natura strettamente letteraria e quasi manierista, e il desiderio di trovare una lingua adatta alla materia trattata finiscono per implodere, mostrando gli ingranaggi con i quali è stata concepita e montata la raccolta. Si tratta di un dittico composto da una strofa di undici versi ( $\left.\mathrm{V}_{\mathrm{Io}}\right)$ :

Me sun scurdâ de mì e l'alegria che m’à ciamâ ai strâd un dì luntan, e sun chì gnèch e û perdü l'arlìa de quèl che me piaseva del vardà, quèl deslassàss de mì nel camenà tra quj cantà d'üsèj ne l'aria svèrsa e curr adré d'i nüver 'm' i bagàj. E'dèss sun chì nascost, tra quèl cialà che fa la vita nel sbassàss di aj, e sòta 'l grév di câ me vègn cun l'aria siren che pàren mort e sensa pas.

Cui risponde, dirimpetto a questa, sulla pagina di destra, una strofa in tutto simile alla prima, ma di quattro versi più lunga $\left(\mathrm{V}_{\mathrm{II}}\right)$ :
Nel smentegàm de mì e la legrìa che m'à ciamâ ai strâd un dì luntan, me sun truà in frosna d'un'arlìa e quèl che me piaseva l'era un can, un feng che te sgagna la cusciensa un gram piasè che te masara i man. Inscì del camenà û pers la sciensa, quèl deslassass de mì nel sgandulà tra quj cantà d'üsej ne l'aria sversa e curr adré aj nüver per giügà.
E'dèss sun chì scundü, tra quj sunaj che surd te fan $j$ urècc e 'l memurià

\footnotetext{
5 «È vero che la donna è un tramite alla vita, che ha un particolare rapporto con le cose carnali e concrete. Ma c'è anche un'altra componente. Quando Dante arriva a vedere Dio, scrive: "All'alta fantasia qui mancò possa". Cioè esprime l'impossibilità di dire l'assoluto. Io dico che Dio è in tutte le cose. Nel rapporto con la realtà sentiamo un cosa - un fiore, una persona, il cielo, le stelle, gli alberi - e magari ci emozionano, però non sappiamo bene cosa sia l'essenza di queste cose. Tale impossibilità di dire è il nascondimento di Dio, come viene definito in teologia. Vorresti dirlo, come per gli occhi di donna, ma non sai come dirli. Allora dici qualcosa attorno, entri appena appena nel senso di questa impossibilità. La poesia, in fondo, è il dire ciò che è impossibile» (Berra 2008).
} 
che crèss la vita nel sbassàss di aj, e sòta el grév di òmm me vègn cun l'aria siren che pàren mort e sensa pas.

Laccostamento, con minimi accorgimenti grafici, delle due ante del dittico darà ragione del metodo con cui ha lavorato il poeta per giungere a questo singolare risultato:

Me sun scurdâ de mì e l'alegria che m'à ciamâ ai strâd un dì luntan, e sun chì gnèch e û perdü l'arlìa de quèl che me piaseva del vardà,

quèl deslassàss de mì nel camenà tra quj cantà d'üsèj ne l'aria svèrsa e curr adré d'i nüver 'm' i bagàj. E'dèss sun chì nascost, tra quèl cialà

che fa la vita nel sbassàss di aj, e sòta 'l grév di câ me vègn cun l'aria siren che pàren mort e sensa pas.
Nel smentegàm de mì e la legrìa che m'à ciamâ ai strâd un dì luntan, me sun truà in frosna d'un'arlìa e quèl che me piaseva l'era un can, un feng che te sgagna la cusciensa un gram piasè che te masara i man. Inscì del camenà û pers la sciensa, quèl deslassass de mì nel sgandulà tra quj cantà d'üsej ne l'aria sversa e curr adré aj nüver per giügà. E'dèss sun chì scundü, tra quj sunaj che surd te fan $j$ urècc e 'l memurià che crèss la vita nel sbassàss di aj, e sòta el grév di òmm me vègn cun l'aria siren che pàren mort e sensa pas.

Un primo innesto di tre versi al v. 4 e un secondo giunto, più breve, ad altezza dell'ottavo (che qui indico in corsivo per maggiore evidenza) trasformano il secondo testo in un isotopo del primo, davvero una copia con varianti dietro la quale si intuisce un modo di procedere, per dilatazioni e riscritture, tipico di molta poesia del Novecento; si pensi a Giorgio Orelli e al suo ciclico ritornare su pochi testi (e temi) cari, sia in poesia che in prosa, ma anche a Montale, a Sereni e alla numerosa schiera dei loro discendenti (nella narrativa l'esempio più eclatante saranno le riscritture ex novo dei romanzi moraviani). Conta molto, in questo caso, che Loi abbia deciso di accostare i due pezzi, i due isotopi, su due pagine affiancate della medesima raccolta, rinunciando alla sostituzione di uno con l'altro, e offrendo invece la genesi, il lavoro attorno al testo, come un risultato degno di attenzione e lettura. È ancora, nel modo più esplicito possibile, un omaggio alla funzione poetica, un elogio della compresenza, di una parola letteraria che si rispecchia in se stessa creando onde inaspettate di senso e di suono.

Detto delle lampanti equivalenze, andranno indagate con attenzione allora le differenze tra i due testi, che lungi dal limitarsi a pochi innesti o al variare di singole tessere (comunque interessanti, soprattutto in ambito 
lessicale: «can» per «vardà», «sgandulà» per «camenà», e simili accorgimenti verso il basso), hanno quale esito principale in sede metrica il passaggio da una forma chiusa $\left(\mathrm{V}_{\mathrm{Io}}\right)$ a una aperta $\left(\mathrm{V}_{\mathrm{II}}\right)$, cioè nient'altro che il convertirsi di una stanza di canzone, di un sonetto o di un madrigale, nel continuum della terzina dantesca ${ }^{6}$. Nel trapasso, apparentemente insignificante, dagli undici versi di $V_{\text {Io }}$ ai quattordici di $V_{\text {II }}$ si richiama insomma alla memoria del lettore un'evoluzione stilistica di grande pregnanza per la letteratura italiana delle origini, che coincise tra l'altro con il superamento, in Dante, dell'esperienza dello Stilnovo e con il definitivo instaurarsi del cantiere della Commedia.

Nello snodo tra i due pezzi gemelli $\mathrm{V}_{\text {Io-}} \mathrm{V}_{\text {II }}$ pare venir meno, in altre parole, quell'entusiasmo manierista con il quale Loi aveva concepito la parte iniziale della raccolta. Uno sguardo alla distribuzione delle parole-chiave lungo tutto l'arco dei 26 pezzi (si confronti la tabella conclusiva) sembrerebbe confermare questa ipotesi, se è vero che la progressiva rinuncia a termini quali pias, ver, dònna, cör o respira lascia spazio, pagina dopo pagina, ad accenti marcatamente più moderni (citâ, finester, trenu) e persino alle parole scurrili (tètt, cü, merda) tipiche della tradizione in vernacolo. La cesura più forte, e stilisticamente irreversibile, si registra all'altezza di $\mathrm{V}_{24} \mathrm{e}$ si conferma nei brani conclusivi («Se spacca el vìv. Vu'n gir e vedi tocch / d'una Milan de gent che va a cassü: / fial e siringh tra l'erba, udur de socch / che taccâ al mür venden figa e cü..», $V_{25}$, vv. I-4), sebbene non manchino già nei primi pezzi avvisaglie di questo lento incedere verso regioni infernali lontane dalla purezza stilnovista: «Sbianca la nott la ghe tegniva in scoss, / e la citâ despersa dré di spall» (VI2, vv. I-2), «Me disen la mural l'û dada ai can. / Sé l'è mural? 'na bestia?'na mattada?» (V $\mathrm{V}_{\mathrm{I}}$, vv. I-2).

Resta da dire del titolo, quell'inverno che ricorre un'unica volta nel libro - «Se sta cume ch'j öcc varda e se spera / 'me l'òm del verna véd el sû nel fàss» ( $\mathrm{V}_{\mathrm{I} 6}$, vv. 9-IO) - e dietro il quale sarà lecito intravvedere il protagonista di un viaggio che, sulle orme di Dante, e senza dimenticare chi lo ha riletto nel Novecento (Montale in primis, ma anche Sereni e Ungaretti), ha portato «l'uomo dell'inverno» Franco Loi a un risultato di notevole valore letterario, che meriterebbe di essere studiato più a fondo di quanto non è stato possibile fare in queste pagine.

6 Il metro della terza rima di matrice dantesca, per il quale andrà fatto ancora una volta, in ambito lombardo, il nome di Carlo Porta, ricorre tre volte in Verna, per lo più nella seconda parte del libro ( $\left.\mathrm{V}_{\mathrm{II}}, \mathrm{V}_{\mathrm{I}} \mathrm{e} \mathrm{V}_{25}\right)$. 


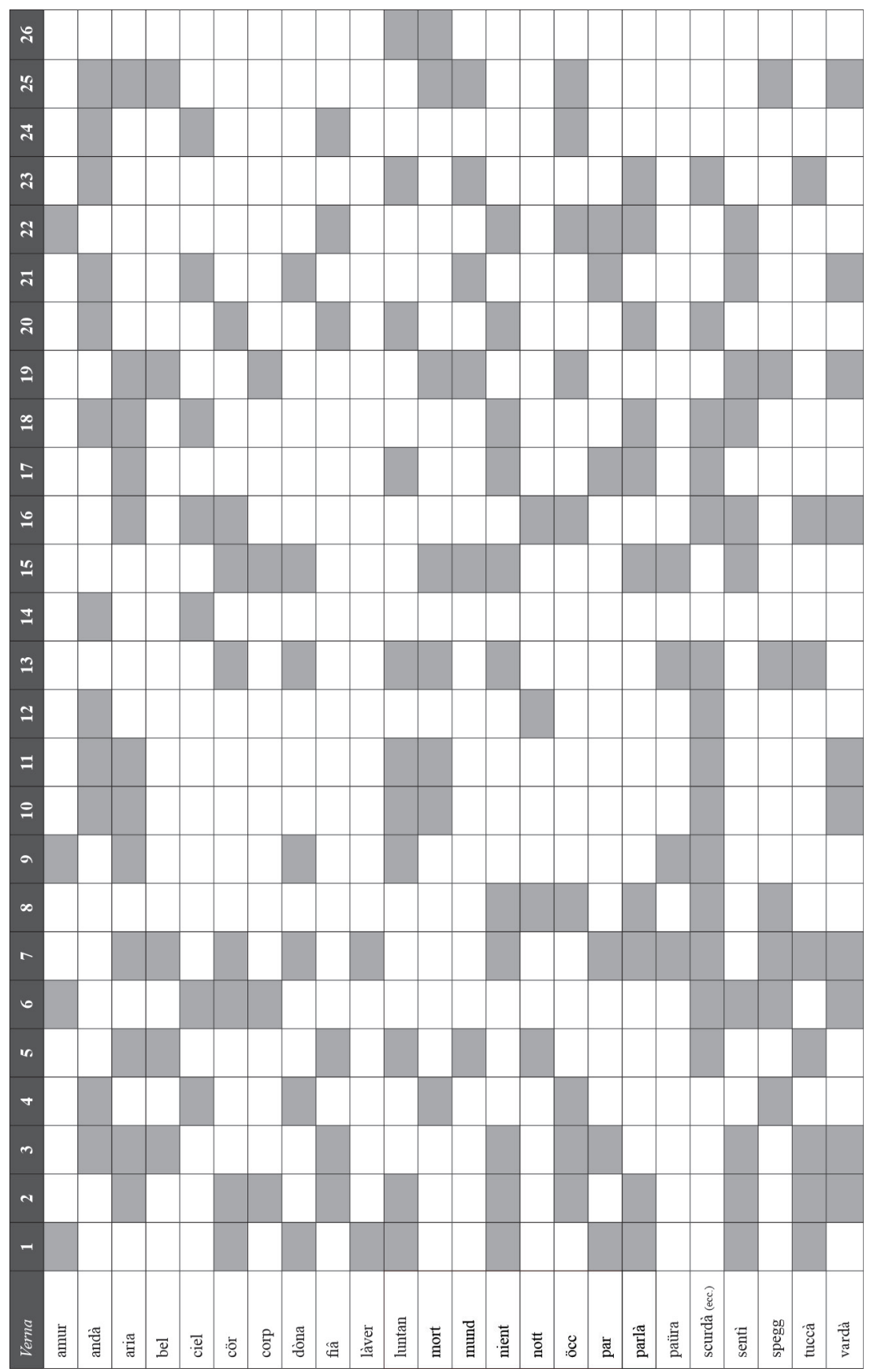




\section{Bibliografia}

Berra, Pietro, «Il poeta Franco Loi : "Dio si racconta solo con gli occhi di una donna"», La Provincia di Como, I6 ottobre 2008.

Contini, Gianfranco, «Esercizio di interpretazione sopra un sonetto di Dante», in Varianti e altra linguistica. Una raccolta di saggi (I938-I968), Torino, Einaudi, I970, pp. I6I-I68; poi anche in Un'idea di Dante. Saggi danteschi, Torino, Einaudi, 1976, pp. 2 I-29.

Loi, Franco, Verna, presentazione di Daniela Attanasio, Roma, Edizioni Empiria («Poesia \& variazioni», I), 1997.

Loi, Franco, Aria de la memoria. Poesie scelte 1973-2002, Torino, Einaudi, 2005.

Loi, Franco, Da bambino il cielo. Autobiografia, a cura di Mauro Raimondi, Milano, Garzanti, 2010.

Porta, Carlo, Poesie, a cura di Dante Isella, nuova edizione rivista e accresciuta, Milano, Mondadori («I Meridiani»), 2000. 